OPEN ACCESS

Edited by:

Antia Botana,

Arizona State University, United States

Reviewed by:

Atsushi Fujimori,

Waseda University, Japan

Franca Manghi,

University of Modena and Reggio

Emilia, Italy

*Correspondence:

Thomas P. Devereaux

tpd@stanford.edu

Specialty section: This article was submitted to

Condensed Matter Physics,

a section of the journal

Frontiers in Physics

Received: 16 December 2021

Accepted: 17 January 2022

Published: 28 February 2022

Citation:

Been EM, Hsu KH, Hu Y, Moritz B, Cui Y, Jia C and Devereaux TP (2022)

On the Nature of Valence Charge and

Spin Excitations via Multi-Orbital

Hubbard Models for Infinite-

Layer Nickelates.

Front. Phys. 10:836959

doi: 10.3389/fphy.2022.836959

\section{On the Nature of Valence Charge and Spin Excitations via Multi-Orbital Hubbard Models for Infinite-Layer Nickelates}

\author{
Emily M. Been ${ }^{1,2}$, Kuan H. Hsu ${ }^{1,3}$, Yi Hu ${ }^{1,3}$, Brian Moritz ${ }^{1}$, Yi Cui ${ }^{3}$, Chunjing $\mathrm{Jia}^{1}$ and \\ Thomas P. Devereaux ${ }^{1,3 *}$
}

${ }^{1} S L A C$ National Accelerator Laboratory, Stanford Institute for Materials and Energy Sciences, Menlo Park, CA, United States, ${ }^{2}$ Department of Physics, Stanford University, Stanford, CA, United States, ${ }^{3}$ Department of Materials Science and Engineering, Stanford University, Stanford, CA, United States

Building upon the recent progress on the intriguing underlying physics for the newly discovered infinite-layer nickelates, in this article we review an examination of valence charge and spin excitations via multi-orbital Hubbard models as way to determine the fundamental building blocks for Hamiltonians that can describe the low energy properties of infinite-layer nickelates. We summarize key results from density-functional approaches, and apply them to the study of $\mathrm{x}$-ray absorption to determine the valence ground states of infinite-layer nickelates in their parent form, and show that a fundamental $d^{9}$ configuration as in the cuprates is incompatible with a self-doped ground state having holes in both $d_{x^{2}-y^{2}}$ and a rare-earth-derived axial orbital. When doped, we determine that the rareearth-derived orbitals empty and additional holes form low spin $(S=0) d^{8} \mathrm{Ni}$ states, which can be well-described as a doped single-band Hubbard model. Using exact diagonalization for a 2-orbital model involving $\mathrm{Ni}$ and rare-earth orbitals, we find clear magnons at 1/2 filling that persist when doped, albeit with larger damping, and with a dependence on the precise orbital energy separation between the $\mathrm{Ni}$ - and rare-earthderived orbitals. Taken together, a full two-band model for infinite-layer nickelates can well describe the valence charge and spin excitations observed experimentally.

Keywords: nickelate, superconductor, exact diagonalization, dynamic spin structure factor, spectroscopy, $x$-ray absorption spectroscopy, XAS

\section{INTRODUCTION}

The discovery of experimental superconductivity in infinite-layer nickelates [1], 20 years after they were theoretically proposed [2], has unveiled new territory to probe the unknowns of unconventional superconductivity. The comparison between the superconducting nickelates to the cuprates has proven to be a rich area of inquiry. Significant progress has been made in the last 2 years, but many mysteries about this novel superconducting family remain unsolved. As pointed out early on [3], the bandstructure for $\mathrm{LaNiO}_{2}$ while being primarily $3 d^{9} \mathrm{Ni}$ near the Fermi level has additional small Fermi pockets of largely axial character involving $\mathrm{Ni} 3 d_{z^{2}}$ and La $5 d_{z^{2}}$ orbitals. Including a Hubbard $U$ on nickel splits the $d^{9}$ states but still leaves itinerant states at the Fermi level, indicating that the parent compound for infinite-layer nickelate is not analogous to undoped $\mathrm{CuO}_{2}$ as an antiferromagnetic Mott insulator. Moreover, the location of the centroid of the oxygen states in 

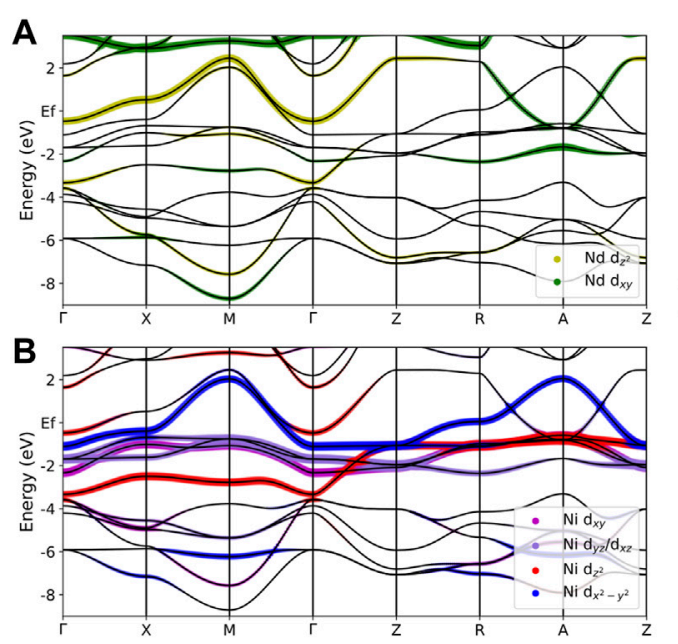

C

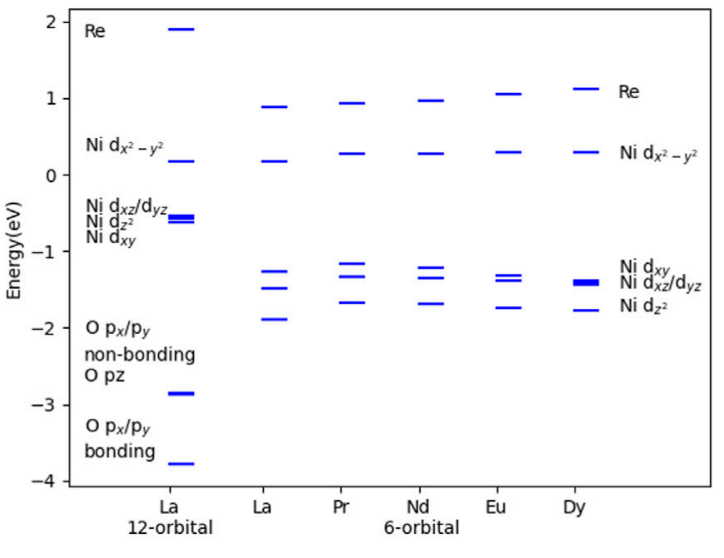

FIGURE 1 | (A) DFT calculated band structure and projected Nd $5 d$ orbital content for infinite-layer nickelate $\mathrm{NdNIO}_{2}$. (B) DFT calculated band structure and projected $\mathrm{Ni} 3 d$ orbital content for $\mathrm{NdNiO}_{2}$. (C) Atomic energy level diagram for infinite-layer nickelates $R \mathrm{NiO}_{2}$, where $R=\mathrm{La}, \mathrm{Pr}, \mathrm{Nd}$, Eu, and Dy. On-site energies are obtained by 6 orbital and 12-orbital Wannier downfolding from DFT calculated band structures. All energy levels are relative to the Fermi level of the corresponding material.

$\mathrm{LaNiO}_{2}$ lies at lower energies than $\mathrm{CuO}_{2}$ compounds, moving them closer to the boundary between Mott-Hubbard systems and charge-transfer insulators in the Zaanen-Sawatzky-Allen scheme [4]. While there is scant evidence that $\mathrm{LaNiO}_{2}$ is antiferromagnetic, resonant inelastic $x$-ray scattering (RIXS) has clearly revealed propagating magnons, yielding a spin exchange energy $\sim 60 \mathrm{meV}$ in the parent compound [5]. These excitations persist when doped, albeit with larger damping [5], in a way that is not too disimilar to paramagnon excitations in doped cuprates [6-8].

Thus there already is a rich amount of information from which an estimate for a fundamental low energy model Hamiltonian can be obtained and used to model the phase diagram of infinite-layer nickelates. In this article we review efforts to combine density functional approaches with cluster exact diagonalization to determine such a model. Specifically we utilize x-ray absorption (XAS) as a tool to determine valence charge states in the undoped and doped infinite-layer nickelates, arriving at a low-energy 2orbital model Hamiltonian. We then perform calculations on finite-sized clusters to determine the dynamic spin structure factor in undoped and doped systems. Our results yield a consistent description of charge valence and spin excitation states in the infinite-layer nickelates, indicating that a 2-orbital model likely contains the fundamental ingredients needed to explore superconductivity and the rich phase diagram in these compounds.

\section{METHODS}

\subsection{Density Functional Theory}

The electronic structure of the infinite-layer nickelates $\mathrm{RNiO}_{2}$ have been evaluated using density functional theory (DFT) in the generalized gradient approximation (GGA) for the exchangecorrelation functional as implemented in Quantum Espresso
$[9,10]$. The band structure near the Fermi energy that was obtained for $\mathrm{NdNiO}_{2}$ is shown in Figures 1A, B.

To obtain a microscopic Hamiltonian and understand the atomic energy levels, we downfolded the bandstructure as implemented in Wannier90 [11] to obtain the tight-binding model parameters $t_{i, j}^{\mu, \nu}$ as shown in Eq. 2 below. Two types of Wannier downfolding have been calculated: (Eq. 1) a 12-orbital model downfolding, which includes five Ni $3 d$ orbitals, one $R 5 d$ orbital and six O $2 p$ orbitals; and (Eq. 2) a 6-orbital model downfolding, which includes five Ni $3 d$ orbitals and one $R 5 d$ orbital. For $\mathrm{NdNiO}_{2}$, the bandstructure corresponding to the 6orbital model spans an energy range from $\sim-3.5 \mathrm{eV}$ to $\sim 2 \mathrm{eV}$, covering the most prominent low energy features. The bands below $\sim-3.5 \mathrm{eV}$ for $\mathrm{NdNiO}_{2}$ have predominantly oxygen orbital content. Figure 1C summarizes the atomic energy level diagram, which shows the on-site energies from the Wannier downfolding for a series of $R \mathrm{NiO}_{2}$ materials ( $R=\mathrm{La}, \mathrm{Pr}, \mathrm{Nd}, \mathrm{Eu}$ or Dy).

\subsection{X-Ray Absorption from Multi-Orbital Hubbard Hamiltonians}

We focus on the Ni $L$-edge $(2 p \rightarrow 3 d)$ XAS utilizing a cluster model for monovalent $\mathrm{NiO}_{2}$ containing five $\mathrm{Ni} 3 d$ orbitals and six O $2 p$ orbitals in a square-planar geometry, and three $\mathrm{Ni} 2 p$ orbitals per unit cell in the atomic core, with hybridization parameters obtained from Wannier downfolding as described in the previous section. We evaluate the XAS $\kappa$ for the absorption of a photon having momentum and polarization $\mathbf{k}_{i}, e_{i}$, respectively, as

$$
\kappa_{\mathbf{e}_{i}, \mathbf{k}_{\mathrm{i}}}(\omega)=\frac{1}{\pi Z} \sum_{i, \nu} e^{-\beta E_{i}}\left|\left\langle\nu\left|\hat{D}_{\mathbf{k}_{i}}\left(\mathbf{e}_{i}\right)\right| i\right\rangle\right|^{2} \delta\left(\omega-\left(E_{\nu}-E_{i}\right)\right),
$$

Here, $Z$ is the partition function, $E_{\{i, v\}},|\{i, v\}\rangle$ are the energies and eigenstates of the initial (ground state) and XAS final states, respectively, obtained from exact diagonalization of the 


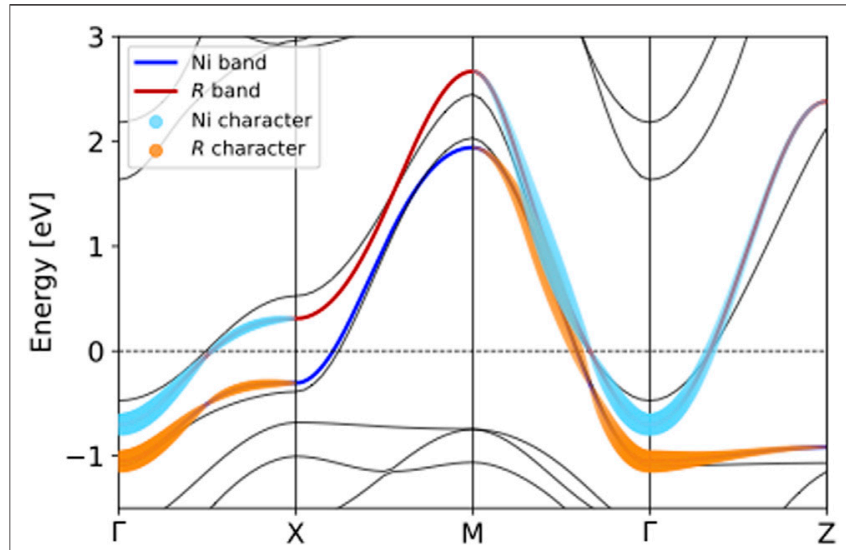

FIGURE 2 | Hybridization of the 2-orbital model for $\mathrm{NdNiO}_{2}$, as described in Eq. 4, showing subdominant $\mathrm{Ni}$ and $R$ band character, respectively.

multi-orbital Hubbard model in the cluster Hilbert space. The multi-orbital Hubbard model can be compactly written as

$$
\begin{aligned}
H & =\sum_{i, j, \sigma} \sum_{\mu, \nu} t_{i, j}^{\mu, \nu} d_{i, \mu, \sigma}^{\dagger} d_{j, v, \sigma} \\
& +\frac{1}{2} \sum_{i} \sum_{\mu, \nu, \mu^{\prime}, \nu^{\prime}} \sum_{\sigma, \sigma^{\prime}} U_{\mu, \nu, \mu^{\prime}, \nu^{\prime}} d_{i, \mu, \sigma}^{\dagger} d_{i, v, \sigma^{\prime}}^{\dagger} d_{i, \mu^{\prime}, \sigma^{\prime}} d_{i, v^{\prime}, \sigma} .
\end{aligned}
$$

Here $i, j$ denote sites, $\sigma, \sigma^{\prime}$ denote spin, and $\mu, \nu, \mu^{\prime}, \nu^{\prime}$ denote orbital indices. The operators $d_{i, \mu, \sigma}^{\dagger}, d_{i, \mu, \sigma}$ create, annihilate holes having spin $\sigma$ in orbital $\mu$ at site $i$, and can represent valence, ligand, or core holes. The Coulomb matrix elements $U$ are written in terms of Slater-Condon parameters pertaining to the valence hole and core hole states, which can be determined via a variety of methods or simply used as fitting parameters to $\mathrm{x}$-ray spectra. Lastly $\hat{D}$ is the dipole operator connecting $\mathrm{Ni} 2 p$ core levels to $\mathrm{Ni} 3 d$ states. Additional details about XAS can be found in various textbooks, i.e. Ref. [12]. As the Hilbert space scales exponentially with the number of orbitals in the unit cell, this technique is amenable to only small clusters. However, since XAS is a local probe of electronic structure, our computational approach is well suited to determine the effective low energy valence and spin states of $\mathrm{Ni}$, as is well known in many other contexts.

\subsection{Dynamic Spin Structure Factor}

The dynamic spin structure factor has been shown to be an accurate approximation of the RIXS cross section of both Mott insulators and doped systems [6, 13]. The dynamical spin structure factor is defined as

$$
S(\mathbf{q}, \omega)=\frac{1}{\pi} \operatorname{Im}\left\langle G\left|\rho_{\mathbf{q}}^{(s) \dagger} \frac{1}{\mathcal{H}-E_{G}-\omega-i \Gamma} \rho_{\mathbf{q}}^{(s)}\right| G\right\rangle,
$$

where the spin density operator is given by $\rho_{\mathbf{q}}^{(s)}=\sum_{\mathbf{i}, \sigma} s_{\mathbf{i} \sigma} e^{i \mathbf{q} \cdot \mathbf{r}_{\mathbf{i}}}=\sum_{\mathbf{k}, \sigma} \sigma c_{\mathbf{k}+\mathbf{q}, \sigma}^{\dagger} c_{\mathbf{k} \sigma}$, and the 2-orbital nickelate model Hamiltonian is given by

$$
\begin{aligned}
\mathcal{H}= & \sum_{\mathbf{k}, \sigma}\left(\varepsilon_{\mathbf{k}}^{R} c_{\mathbf{k} \sigma}^{\dagger} c_{\mathbf{k} \sigma}+\varepsilon_{\mathbf{k}}^{\mathrm{Ni}} d_{\mathbf{k} \sigma}^{\dagger} d_{\mathbf{k} \sigma}\right)+\frac{U}{N} \sum_{\mathbf{k}_{1}, \mathbf{k}_{2}, \mathbf{q}} d_{\mathbf{k}_{1}+\mathbf{q}, \uparrow}^{\dagger} d_{\mathbf{k}_{2}-\mathbf{q}, \downarrow}^{\dagger} d_{\mathbf{k}_{2}, \downarrow} d_{\mathbf{k}_{1}, \uparrow} \\
& +\sum_{\mathbf{k}, \sigma}\left(\varepsilon_{\mathbf{k}}^{R-N i} c_{\mathbf{k}, \sigma}^{\dagger} d_{\mathbf{k}, \sigma}+\text { H.c. }\right),
\end{aligned}
$$

where $c_{\mathbf{k}}\left(c_{\mathbf{k}}^{\dagger}\right)$ operators represent the dispersive $R 5 d$ band and the $d_{\mathbf{k}}\left(d_{\mathbf{k}}^{\dagger}\right)$ operators represent the Hubbard-like Ni $3 d$ band. The details of $\varepsilon_{\mathbf{k}}^{R}, \varepsilon_{\mathbf{k}}^{\mathrm{Ni}}$, and $\varepsilon_{\mathbf{k}}^{\mathrm{R}-\mathrm{Ni}}$ are defined in Ref. [14] which proposed this form of the 2-orbital model. The hybridization between $\mathrm{Ni}$ and $R$ in this 2-orbital model is shown visually by plotting only the subdominant orbital character in Figure 2. This hybridization results in the "self-doped" nature of the nickelates.

We evaluate the dynamic spin structure factor, $S(\mathbf{q}, \omega)$, for the 2-orbital nickelate model using exact diagonalization (ED) on an 8-site (diamond-shaped) Betts cluster [15] with periodic boundary conditions, which suffers from finite-size effects but is sufficient for determining the magnon spectrum on the antiferromagnetic Brillouin zone boundary at $(\pi / 2, \pi / 2)$. We take the value of the Hubbard $U=8 \mathrm{eV}$ so that we get a reasonable estimate of $J \sim 80 \mathrm{meV}$. The eigenvalues and eigenvectors of the ED calculations were found using the Implicitly Restarted Lanczos Method from the ARnoldi PACKage (ARPACK), as implemented in SciPy Linalg library ([16], RRID: SCR_008058). The biconjugate gradient stabilized method was used to calculate $S(\mathbf{q}, \omega)$.

\section{RESULTS}

\subsection{X-Ray Absorption Spectroscopy \\ 3.1.1 Single-Site}

The single site XAS is simulated using techniques mentioned in Sec. 2.2, where the Hilbert space is determined using only the $3 d$ (valence) and $2 p$ (core) orbitals of the Ni transition metal ion, without ligand $2 p$ orbitals, and with open boundary conditions. The eigenenergies are obtained by diagonalizing the Hamiltonian and the XAS cross section is evaluated using Eq. (1).

We first calculate the multiplet XAS of the $d^{9}$ electronic configuration of the $\mathrm{Ni}$ ion where the Slater-Condon, spin orbit coupling and crystal-field splitting parameters are taken from Ref. [17]. The results are plotted in Figure 3. Since the single site calculation does not include ligand orbitals in the Hilbert space, direct measurements of crystal field splitting of $d$ orbitals energy levels are used without hybridization parameters obtained by Wannier downfolding from Ref. [18]. Although hybridization with ligands is not considered in this single-site calculation, it can still capture the difference in XAS between high-spin and low-spin ground state as described below. The Coulomb interaction for $d^{9}$ ions is less relevant and the spectral lineshape is dominated by the spin orbit coupling of the core levels, showing a single peak for both the $L_{3}$ and $L_{2}$ edges with light polarization along the $\mathrm{x}$-direction, and no absorption with light polarization along the $z$-direction, due to the orbital $d_{x^{2}-y^{2}}$ character of the holes in the $d^{9}$ configuration. We then 


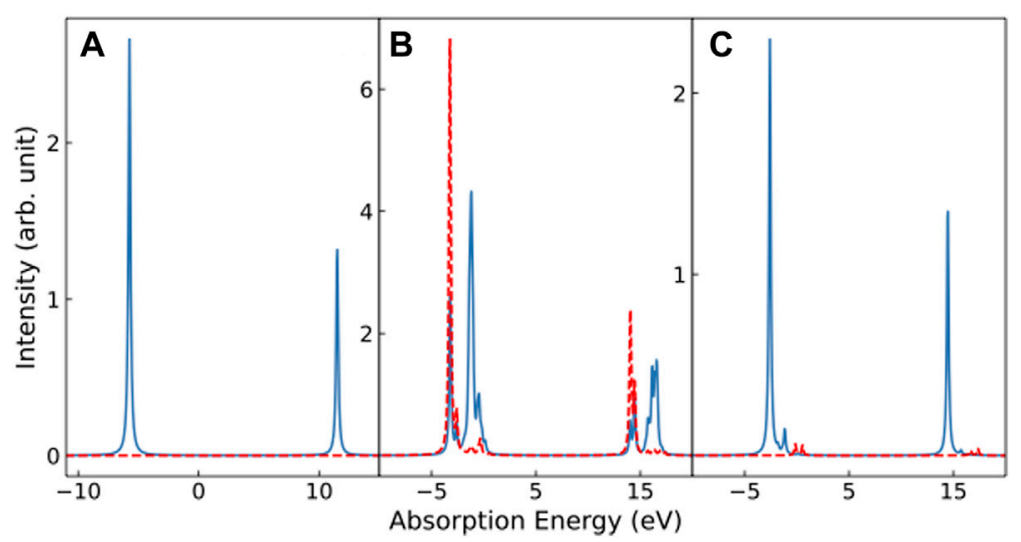

FIGURE 3| Multiplet XAS calculations for the single-site nickel ion. The absorption energy is measured with respect to the ground state of $d^{9}$ ion. The solid line is the spectra measured with $x$-polarized light, and the dashed line is the spectra measured with $z$-polarized light. From left to right are spectra of (A) the $d^{9}$ ion, (B) the $d^{8}$ highspin ion, and (C) the $d^{8}$ low-spin ion.

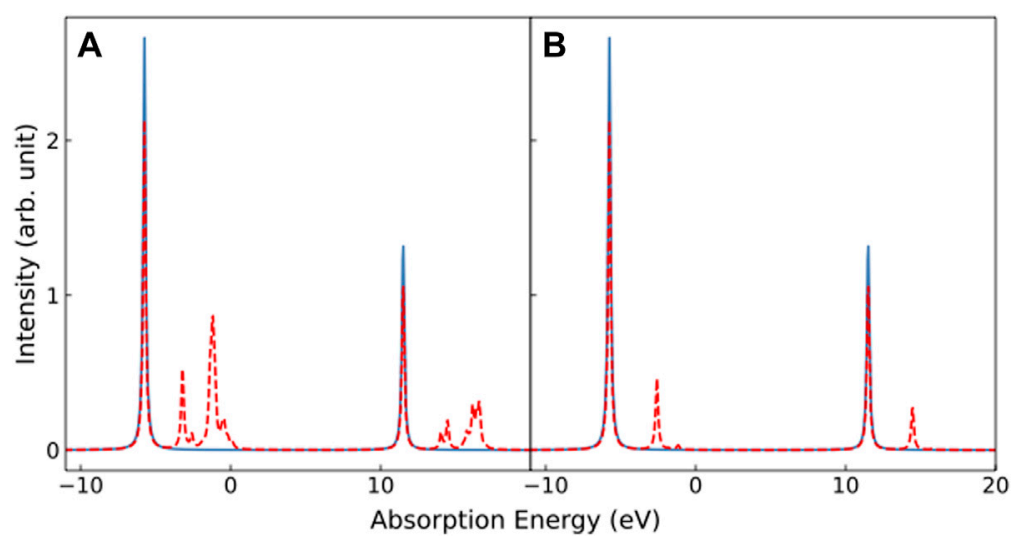

FIGURE 4 |XAS spectra for the undoped $d^{9}$ ion (solid line) and with $20 \%$ of the hole-doped $d^{8}$ ion (dashed line) calculated for $x$-polarization. Panel (A) shows the results for doping with the $d^{8}$ high-spin state, and panel $\mathbf{B}$ ) shows the results for doping with the $d^{8}$ low-spin state.

calculated the multiplet XAS on the $d^{8}$ ion using the same parameters for a spin singlet $(S=0)$ and a spin triplet $(S=1)$ ground state. Similar to previous reports by Ref. [17], with light polarized along the $z$-direction, the intensity of the XAS peaks decrease in the low spin state, while the intensity remains the same order of magnitude for the high spin state. With light polarized along the $x$-direction, the highspin ground state displays stronger intensity across a wide range of absorption energies, with more dipole-allowed transitions to excited states, compared to the singly degenerate, low-spin ground state.

We also simulated doped spectra as a linear combination of the $d^{8}$ and $d^{9}$ spectra, with the results plotted in Figure 4. Clearly, the high-spin $d^{8}$ state produces spectra with a wide energy spread and additional multiplet peaks. A better comparison to experiment [17] can be made if the $d^{8}$ ion is in the low-spin configuration, producing a less pronounced shoulder in addition to the main absorption peak from the dominant $d^{9}$ configuration.

\subsubsection{Two-Site}

The two-site XAS is simulated with a two-site, multi-orbital Hubbard model as described in Sec. 2.2 with periodic boundary conditions in contrast to section Sec. 3.1.1, with 11 orbitals for each site (unit cell $\mathrm{NiO}_{2}$ ) in the initial state that includes six oxygen $2 p$ orbitals and the five nickel $3 d$ orbitals, and 14 orbitals for one of the cells in the final state, which includes an additional three core-level nickel $2 p$ orbitals for the target atom. The eigenenergies and eigenstates are obtained by exact diagonalization of the model Hamiltonian and the XAS spectra are calculated using Eq. (1). Including the oxygen ligand orbitals in the model allows hybridization and charge transfer effects between oxygen $2 p$ and Nickel $3 d$ orbitals. The Ni-O hybridization will affect not only the effective energy levels of the Nickel $3 d$ orbitals, but this also changes the effective band character, with oxygen ligand $p$ orbital contributions to the valance and conduction bands, which will result in changes to the XAS lineshape compared to the single-site calculation in Sec. 3.1.1. 


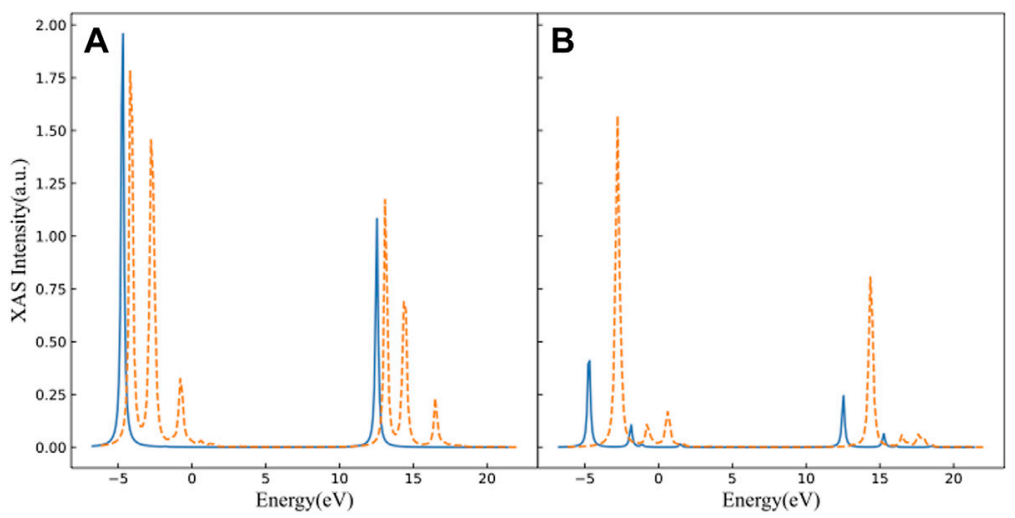

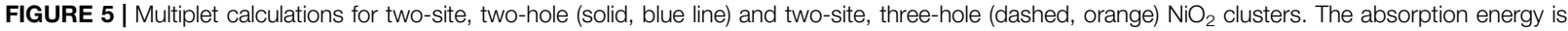
measured from the ground state of the two-site, two-hole calculation. Panel (A) shows the XAS spectra in $x$-polarization; and panel (B) shows the XAS spectra in z-polarization.

\section{A}

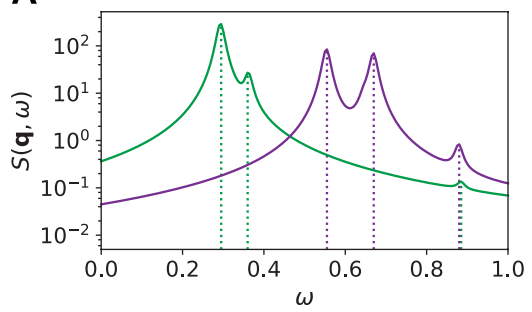

B

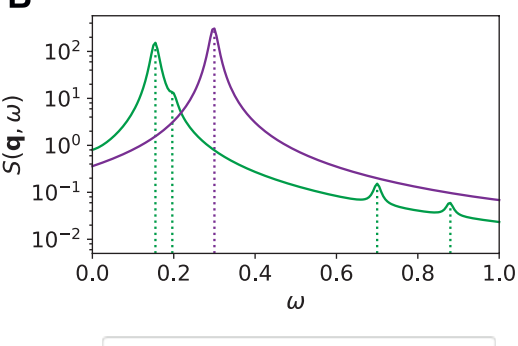

C

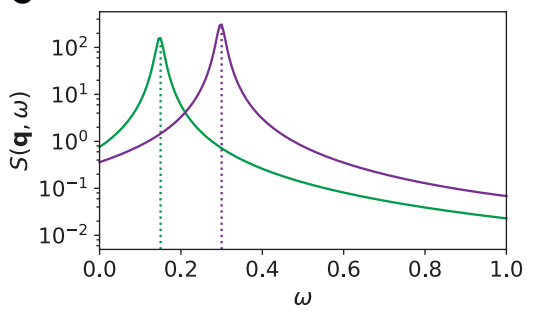

FIGURE 6 | Comparison of $S(\mathbf{q}, \omega)$ at $\mathbf{q}=\left(\frac{\pi}{2}, \frac{\pi}{2}\right)$ between $(\mathbf{A})$ the 2-orbital model with $\varepsilon_{\mathbf{k}}^{R}-\varepsilon_{\mathbf{k}}^{N i} \equiv \Delta \sim 1$ eV, (B) the 2-orbital model with $\Delta \rightarrow \Delta+U / 2$, and $(\mathbf{C})$ the 1 -orbital model that only includes the Ni layer. The dotted vertical lines in each plot show positions of energy eigenvalues (differences) broadened by Lorentzian convolution in the final spectrum. All energies are in $\mathrm{eV}$ with $\mathbf{S}(\mathbf{q}, \omega)$ shown on a log scale to enhance weaker features. The half-filling curve (green) in panel (A) aligns with the doped curves (purple) in panels (B) and (C) which correspond to $25 \%$ doping of the nickel layer, highlighting the "self-doping" influence of the rare-earth layer when $\Delta$ is small.

We performed multiplet calculations for a two-site, two-hole (nominally $d^{9}$ nickel) and a two-site, three-hole (doped $\mathrm{NiO}_{2}$ planes) cluster using effective parameters described in Sec. 3.1.1, and hopping parameters $\left(t_{p d}, t_{p d z}, t_{p d x y}, t_{p d x z}, t_{p d y z}\right.$ are 1.2, $0.3,0.7,0.8$ and $0.8 \mathrm{eV}$, respectively) obtained by Wannier downfolding as described in Sec. 2.1. The inclusion of hybridization parameters allow us to modulate the strength of electron hopping between each orbital and the onsite energy, with the electrons distributed between the different orbitals based on the model Hamiltonian as described in Ref. [18]. These XAS spectra are shown in Figure 5. The two-site, two-hole XAS spectrum with $x$-polarization is similar to the single-site, $d^{9}$ model, which was described in the previous section. When doped with one hole, the $L_{3}$ and $L_{2}$ edges split into three peaks due to Ni-O hybridization. These results are consistent with both the single-site calculation and the experimental results [17].

\subsection{Spin Structure Factor}

Results for the dynamical spin structure factor calculated via exact diagonalization are plotted in Figure 6, showing a comparison of the 2-orbital model with two different values of the energy difference between $\mathrm{Ni}$ and $R$ orbitals $\varepsilon_{\mathbf{k}}^{R}-\varepsilon_{\mathbf{k}}^{N i} \equiv \Delta$, and also results from a single-orbital model for the nickel oxide layer. "Half-filling" corresponds to eight electrons distributed among the 16 orbitals of the 2-orbital, $8 \mathrm{~A}$ Betts cluster, or the eight orbitals of the $8 \mathrm{~A}$ Betts cluster in the 1-orbital model for the Ni-O layer. The Ni-R energy difference and hybridization dictate the effective filling of the $\mathrm{Ni}$ band in the 2-orbital model. The doped calculation is performed with six electrons, corresponding to a nominal doping of $~ 25 \%$.

For the 2-orbital model, tuning the energy difference between $\mathrm{Ni}$ and $R$ sites, $\Delta$, plays a major role in the occupation of the $R$ orbital, and hence the "self-doping" of the Ni layer. In Figure 6A, $\Delta \sim 1 \mathrm{eV}$ results in approximately two electrons occupying the $R$-sites at both overall half-filling and $\sim 25 \%$ doping, meaning the $\mathrm{Ni}$ layer retains a $25 \%$ "self-doping" offset. In contrast, panel Figure 6B shows the results for the 2-orbital model with a large offset of $U / 2=4 \mathrm{eV}$ added to $\Delta$, resulting in $\Delta \sim 5 \mathrm{eV}$. Here, the $R$-site is almost completely empty and $S(\mathbf{q}, \omega)$ looks very similar to results from the 1-orbital calculation shown in Figure 6C. 
Obviously, the infinite-layer nickelates live between these two extremes and Figure 6 gives us some clues as to how $S(\mathbf{q}, \omega)$ is affected by the occupation of the $R$ sites, with $S(\mathbf{q}, \omega)$ extremely sensitive to the $R$ and Ni occupations.

\section{DISCUSSION}

In this paper we explored numerical simulations using DFT and $\mathrm{ED}$ to determine the underlying band structure, valence configuration, and dynamical spin response of model Hamiltonians aimed to describe infinite-layer nickelates. DFT for various $R$ substituted nickelates was used to determine the parameters of an effective 2-orbital model including Ni $3 d_{x^{2}-y^{2}}$ and $R 5 d$ "axial" orbitals, where the effect of lower-lying oxygen orbitals is to modify the inter-orbital hybridizations. Cluster multiplet ED was used to determine that undoped nickelate has predominantly $3 d^{9}$ valence which upon doping involves low-spin nickel $3 d^{8}$ valence holes. These results indicate that undoped infinite-layer nickelate is "self-doped" away from halffilling $\mathrm{Ni} 3 d^{9}$ via the presence of a finite electron concentration in the $R$ layer compensating the holes in the Ni layer, the physics of doped nickelates may be described simply from the point of view of a one-band Hubbard-like model. Our consideration of the spin response indicates a close similarity of the paramagnon energies and intensities to one-band systems, in close analogy with the behavior seen in the cuprates.

Our ED calculations are limited to small clusters and therefore the effect of long-wavelength physics remains beyond our level of investigation. This may be particularly relevant to a discussion of the root cause of superconductivity and the competition between superconductivity and other intertwined phases, such as spin and/or charge stripes that are prevalent in both cuprate phase diagrams and numerical simulations of the single-band Hubbard model [19, 20]. Reference [21] reports density matrix renormalization group (DMRG) simulations of a similar 2-orbital model, and finds Luther-Emery behavior - coexistence of long-range superconducting and charge-density wave order - away from half-filling as in the

\section{REFERENCES}

1. Li D, Lee K, Wang BY, Osada M, Crossley S, Lee HR, et al. Superconductivity in an Infinite-Layer Nickelate. Nature (2019) 572:624-7. doi:10.1038/s41586-019-1496-5

2. Anisimov VI, Bukhvalov D, Rice TM. Electronic Structure of Possible Nickelate Analogs to the Cuprates. Phys Rev B (1999) 59:7901-6. doi:10. 1103/PhysRevB.59.7901

3. Lee K-W, Pickett WE. Infinite-layerLaNiO2: Nil+is notCu2+. Phys Rev B (2004) 70:165109. doi:10.1103/PhysRevB.70.165109

4. Zaanen J, Sawatzky GA, Allen JW. Band Gaps and Electronic Structure of Transition-Metal Compounds. Phys Rev Lett (1985) 55:418-21. doi:10.1103/ PhysRevLett.55.418

5. Lu H, Rossi M, Nag A, Osada M, Li DF, Lee K, et al. Magnetic Excitations in Infinite-Layer Nickelates. Science (2021) 373:213-6. doi:10.1126/science.abd7726

6. Jia CJ, Nowadnick EA, Wohlfeld K, Kung YF, Chen C-C, Johnston S, et al. Persistent Spin Excitations in Doped Antiferromagnets Revealed by Resonant Inelastic Light Scattering. Nat Commun (2014) 5:3314. doi:10.1038/ncomms4314
1D Hubbard model, while the undoped model does not contain long-range antiferromagnetic order, different than single-band Hubbard and more closely in-line with infinitelayer nickelates. The spin dynamics may be investigated using t-DMRG or other techniques, such as determinant quantum Monte Carlo [22]. This remains a topic of future interest.

\section{DATA AVAILABILITY STATEMENT}

The original contributions presented in the study are included in the article/Supplementary Material, further inquiries can be directed to the corresponding author.

\section{AUTHOR CONTRIBUTIONS}

EB performed ED calculations for $S(q, \omega), \mathrm{YH}$ and $\mathrm{KH}$ performed ED calculations for XAS, CJ and EB performed DFT calculations for the nickelate bandstructure. BM, CJ, YC, and TD conceived the project. All authors contributed to the writing of the manuscript.

\section{FUNDING}

This work was supported by the U.S. Department of Energy (DOE), Office of Basic Energy Sciences, Division of Materials Sciences and Engineering. Computational work was performed on the Sherlock cluster at Stanford University and on resources of the National Energy Research Scientific Computing Center, supported by the U.S. DOE, Office of Science, under Contract no. DE-AC02-05CH11231.

\section{ACKNOWLEDGMENTS}

We would like to thank Yao Wang from Clemson University for helpful discussions about exact diagonalization and the 2-orbital nickelate model.

7. Le Tacon M, Ghiringhelli G, Chaloupka J, Sala MM, Hinkov V, Haverkort MW, et al. Intense Paramagnon Excitations in a Large Family of High-Temperature Superconductors. Nat Phys (2011) 7:725-30. doi:10.1038/nphys2041

8. Dean MPM, Dellea G, Springell RS, Yakhou-Harris F, Kummer K, Brookes $\mathrm{NB}$, et al. Persistence of Magnetic Excitations in $\mathrm{La} 2-\mathrm{xSrxCuO} 4$ from the Undoped Insulator to the Heavily Overdoped Non-superconducting Metal. Nat Mater (2013) 12:1019-23. doi:10.1038/nmat3723

9. Giannozzi P, Baseggio O, Bonfà P, Brunato D, Car R, Carnimeo I, et al. QuantumESPRESSO toward the Exascale. J Chem Phys (2020) 152:154105. doi:10.1063/5.0005082

10. Perdew JP, Burke K, Ernzerhof M. Generalized Gradient Approximation Made Simple. Phys Rev Lett (1996) 77:3865-8. doi:10.1103/PhysRevLett. 77.3865

11. Mostofi AA, Yates JR, Pizzi G, Lee Y-S, Souza I, Vanderbilt D, et al. An Updated Version of Wannier90: A Tool for Obtaining Maximally-Localised Wannier Functions. Comp Phys Commun (2014) 185:2309-10. doi:10.1016/j.cpc.2014.05.003

12. de Groot F, Kotani A. Advances in Condensed Matter Science. Boca Raton: CRC Press (2008). doi:10.1201/9781420008425 Core Level Spectroscopy of Solids. 
13. Jia C, Wohlfeld K, Wang Y, Moritz B, Devereaux TP. Using RIXS to Uncover Elementary Charge and Spin Excitations. Phys Rev X (2016) 6:021020. doi:10. 1103/PhysRevX.6.021020

14. Been E, Lee W-S, Hwang HY, Cui Y, Zaanen J, Devereaux T, et al. Electronic Structure Trends across the Rare-Earth Series in Superconducting InfiniteLayer Nickelates. Phys Rev X (2021) 11:011050. doi:10.1103/physrevx.11. 011050

15. Betts DD, Lin HQ, Flynn JS. Improved Finite-Lattice Estimates of the Properties of Two Quantum Spin Models on the Infinite Square Lattice. Can J Phys (1999) 77:353-69. doi:10.1139/p99-041

16. Virtanen P, Gommers R, Oliphant TE, Haberland M, Reddy T, Cournapeau D, et al. SciPy 1.0: Fundamental Algorithms for Scientific Computing in Python. Nat Methods (2020) 17:261-72. doi:10.1038/s41592-019-0686-2

17. Rossi M, Lu H, Nag A, Li D, Osada M, Lee K, et al. Orbital and Spin Character of Doped Carriers in Infinite-Layer Nickelates. Phys Rev B (2021) 104:L220505. doi:10.1103/PhysRevB.104.L220505

18. Hepting M, Li D, Jia CJ, Lu H, Paris E, Tseng Y, et al. Electronic Structure of the Parent Compound of Superconducting Infinite-Layer Nickelates. Nat Mater (2020) 19:381-5. doi:10.1038/s41563-019-0585-z

19. Arovas DP, Berg E, Kivelson S, Raghu S. The Hubbard Model (2021). arXiv e-prints , arXiv:2103.12097.

20. Huang EW, Wang WO, Ding JK, Liu T, Liu F, Huang X-X, et al. Intertwined States at Finite Temperatures in the Hubbard Model. J Phys Soc Jpn (2021) 90: 111010. doi:10.7566/JPSJ.90.111010
21. Peng C, Jiang H-C, Moritz B, Devereaux TP, Jia C. Superconductivity in a Minimal Two-Band Model for Infinite-Layer Nickelates (2021). arXiv e-printsarXiv:2110.07593.

22. Liu F, Peng C, Huang EW, Moritz B, Jia C, Devereaux T (2021). Spin excitations and stripes in the failed antiferromagnetic infinite-layer nickelates. unpublished

Conflict of Interest: The authors declare that the research was conducted in the absence of any commercial or financial relationships that could be construed as a potential conflict of interest.

Publisher's Note: All claims expressed in this article are solely those of the authors and do not necessarily represent those of their affiliated organizations, or those of the publisher, the editors and the reviewers. Any product that may be evaluated in this article, or claim that may be made by its manufacturer, is not guaranteed or endorsed by the publisher.

Copyright (C) 2022 Been, Hsu, Hu, Moritz, Cui, Jia and Devereaux. This is an openaccess article distributed under the terms of the Creative Commons Attribution License (CC BY). The use, distribution or reproduction in other forums is permitted, provided the original author(s) and the copyright owner(s) are credited and that the original publication in this journal is cited, in accordance with accepted academic practice. No use, distribution or reproduction is permitted which does not comply with these terms. 